\title{
Effect of the Jahn-Teller Distortion on Double Exchange Interaction in $\mathrm{La}_{0.8} \mathrm{~K}_{0.2} \mathrm{MnO}_{3}$ Nanoparticles
}

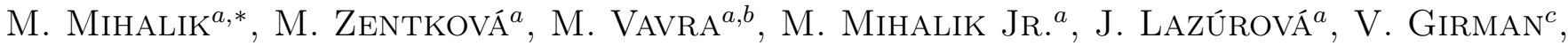 \\ P. KONIECZNY ${ }^{d}$, M. FitTA ${ }^{d}$ AND S. IL'KOVIČ $\check{C}^{e}$ \\ ${ }^{a}$ Institute of Experimental Physics SAS, Watsonova 47, 04001 Košice, Slovakia \\ ${ }^{b}$ Institute of Chemistry, Faculty of Science, P.J. Šafarik University, Moyzesova 11, 04154 Košice, Slovakia \\ ${ }^{c}$ Institute of Physics, Faculty of Science, P.J. Šafarik University, Moyzesova 11, 04154 Košice, Slovakia \\ ${ }^{d}$ Institute of Nuclear Physics, Polish Academy of Sciences, E. Radzikowskiego 152, 31-342 Kraków, Poland \\ ${ }^{e}$ Faculty of Humanities and Natural Sciences, University of Prešov, 17 novembra 1, Prešov, Slovak Republic
}

Electric resistance and effect of hydrostatic pressure on magnetic properties has been studied on the $\mathrm{La}_{0.8} \mathrm{~K}_{0.2} \mathrm{MnO}_{3}$ nanoparticles. Magnetic phase transition is affected by pressure only slightly, the Curie temperature $T_{\mathrm{C}}$ decreases with the rate of $-1.02 \mathrm{~K} / \mathrm{GPa}$, on samples with orthorhombic structure where the Jahn-Teller distortion of lattice is large. On the other hand, $T_{\mathrm{C}}$ increases with the rate of $20.1 \mathrm{~K} / \mathrm{GPa}$ on samples with rhombohedral structure, where the Jahn-Teller distortion of lattice is absent. Insulator type of electrical resistance is characteristic feature of sample with large Jahn-Teller distortion of lattice and insulator-metal transition was observed on samples where the Jahn-Teller distortion is negligible. Our results are in line with theoretical calculation predicting that double exchange interaction is suppressed by the Jahn-Teller distortion.

DOI: 10.12693/APhysPolA.131.875

PACS/topics: 75.50.-y, 75.47.Lx, 75.30.--m, 75.30.Kz

\section{Introduction}

The mixed-valence manganese oxides of the general formula $\mathrm{La}_{1-x} \mathrm{~A}_{x} \mathrm{MnO}_{3}$ (A is a divalent ion like Ca, Sr, $\mathrm{Ba}$, and $\mathrm{Pb}$ ) are a subject of interest due to a desire to understand and exploit the large negative magnetoresistance and magnetocaloric effects [1]. Recently, the same type of manganites doped with univalent metals, such as $\mathrm{Ag}, \mathrm{K}$, and $\mathrm{Na}$ become of great interest, because their physical properties are very sensitive to a magnetic field at room temperature. Group of $\mathrm{La}_{1-x} \mathrm{~K}_{x} \mathrm{MnO}_{3}$ manganites provides a series of new oxides to study magnetocaloric effect $[2,3]$ and insulator-to-metal transition [4] at room temperature.

Newly [5] we have studied magnetic properties of $\mathrm{La}_{0.8} \mathrm{~K}_{0.2} \mathrm{MnO}_{3}$ on nanoparticles, which were prepared by glycine-nitrate method. Crystal structure and particles size were modified by heat treatment. We have found that the crystal structure changes from orthorhombic (space group Pnma) to rhombohedral (space group $R$-3c) after annealing at $600^{\circ} \mathrm{C} / 2 \mathrm{~h} . \mathrm{MnO}_{6}$ - the building blocks of crystal structures are distorted and tilted. We attributed the distortion of the lattice mainly to the Jahn-Teller (JT) distortion, the JT distortion is gradually removed from the sample and completely vanishes in the rhombohedral crystal structure. Scanning electron microscopy (SEM) method revealed that the average size of particle varied with annealing from about $30 \mathrm{~nm}$ to

*corresponding author; e-mail: mihalik@saske.sk
$135 \mathrm{~nm}$. The Curie temperature $T_{\mathrm{C}}$ and the saturated magnetization $\mu_{s}$ increased with annealing and $T_{\mathrm{C}}$ was nearly doubled in the sample with the rhombohedral crystal structure [5]. The exchange bias effect was observed on samples with particles size smaller than $60 \mathrm{~nm}[5]$.

In this work we study the effect of Jahn-Teller (JT) distortion on double exchange interaction in this system. It was shown theoretically that the JT coupling drastically reduces the Anderson-Hasegawa double exchange (DE) [6] and our present work together with the previous results [5] probes the extent of this theoretical result on the $\mathrm{La}_{0.8} \mathrm{~K}_{0.2} \mathrm{MnO}_{3}$ system of nanoparticles experimentally.

\section{Experimental details}

The preparation of nanoparticles followed the glycinenitrate method, where glycine was used as a fuel and nitrates as oxidants [7] and the size of particles was modified by the annealing. The detailed description of annealing procedure, X-ray and SEM analysis is present in [5]. An additional characterisation of prepared sample was performed by transmission electron microscopy (TEM) using a scanning transmission electron microscope JEOL JEM 2100F UHR. Microscope operates with accelerating voltage $80 \mathrm{kV}$ to $200 \mathrm{kV}$ and also enables structure observations at atomic level (HRTEM). Colloids, mixture of the sample and acetone, were prepared for TEM investigation and subsequently a droplet of the colloid was placed on a grid covered by carbon. These particles were covered by carbon layer in next step. Magnetization measurements were performed by a SQUID (MPMS XL-5) in the temperature range from $1.8 \mathrm{~K}$ to $380 \mathrm{~K}$ and in mag- 
netic field up to $5 \mathrm{~T}$. In the case of measurements under pressure the hydrostatic pressure up to $1.4 \mathrm{GPa}$ was generated by a piston cylinder type of the $\mathrm{CuBe}$ pressure cell for MPMS based on Teflon container (Easy Lab). A mixture of mineral oils served as hydrostatic pressure transmitting medium. Resistivity measurements were performed in the temperature range from $300 \mathrm{~K}$ to $50 \mathrm{~K}$ in VERSALAB equipment.

\section{Results and discussion}

TEM study confirmed that all samples consist from agglomerates of nanoparticles as it is shown for sample annealed at $300^{\circ} \mathrm{C}$ for $2 \mathrm{~h}$ (Fig. 1). The size of particles depends on annealing and corresponds with size of nanoparticles determined by scanning electron microscopy [5]. The smallest particles have size approximately from 10 to $15 \mathrm{~nm}$ and typically have character of single crystals as it was revealed by HRTEM study (Fig. 2).

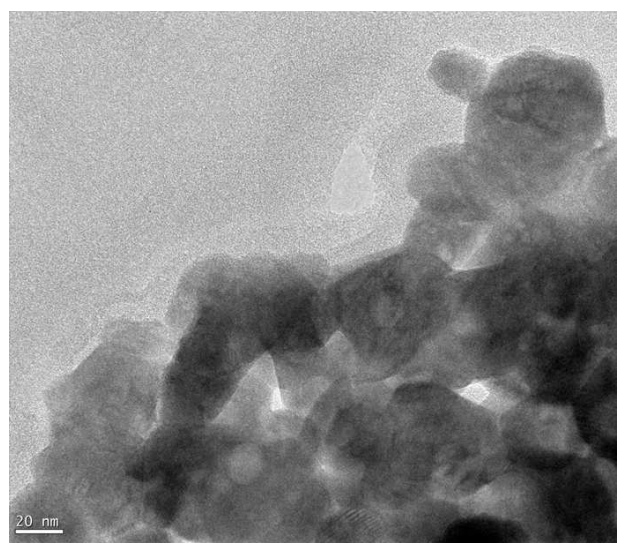

Fig. 1. TEM image shows an agglomerate of nanoparticles. The white line represents the distance of $20 \mathrm{~nm}$.
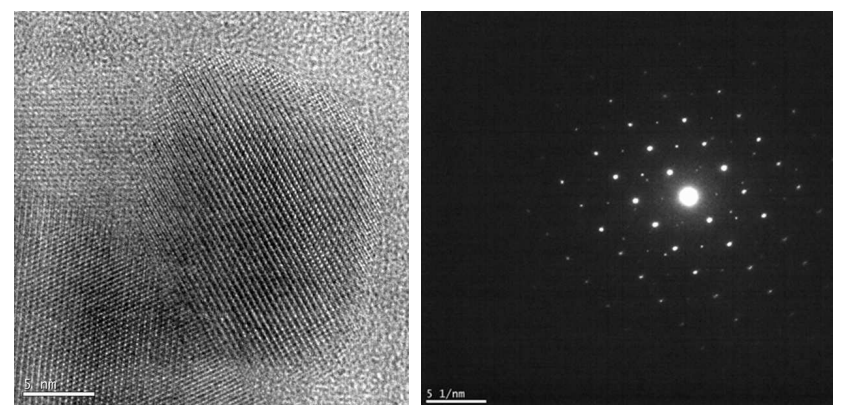

Fig. 2. HRTEM image of as prepared sample shows an individual particle. The white line represents the distance of $5 \mathrm{~nm}$. Second photo shows the electron diffraction pattern.

Hysteretic behaviour between temperature dependence of magnetization in zero field-cooling (ZFC) and fieldcooling (FC) regimes is a characteristic feature of the system with the orthorhombic crystal structure (see Fig. 3). Very large difference between magnetization ZFC and FC curves can indicate structural disorder. Together with

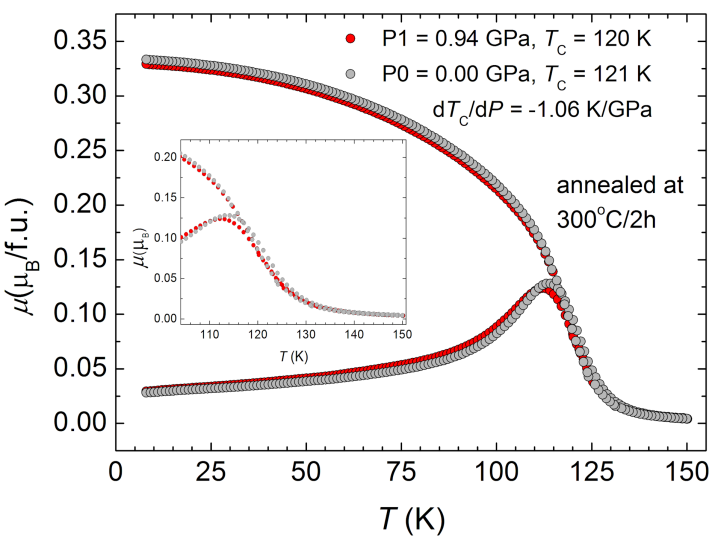

Fig. 3. ZFC and FC magnetization are shown at different pressures for sample with the orthorhombic crystal structure. The inset shows bifurcation and phase transition in detail.

our X-rays diffraction measurements [5] and in analogy with study, which was performed on $\mathrm{La}_{0.7} \mathrm{Ca}_{0.3} \mathrm{MnO}_{3}$ system of nanoparticles [8], we suppose that the JT distortion of crystal lattice is mainly responsible for this disorder. Pressure effect on the Curie temperature $T_{\mathrm{C}}$ is very small and even $T_{\mathrm{C}}$ decreases with pressure with rate $-1.06 \mathrm{~K} / \mathrm{GPa}$ (see Fig. 3). Such behaviour is unusual for hole-doped manganites with dominant DE interaction, where pressure stabilises ferromagnetic (FM) ordering and increases $T_{\mathrm{C}}[9]$. It was shown theoretically that the JT coupling drastically reduces the AndersonHasegawa double exchange (DE) [6] and in this context our pressure measurements correspond with theoretical prediction [6]. We associate small increase of $T_{\mathrm{C}}$, i.e. the increase of FM interactions with annealing [5], with reduction of JT distortions.

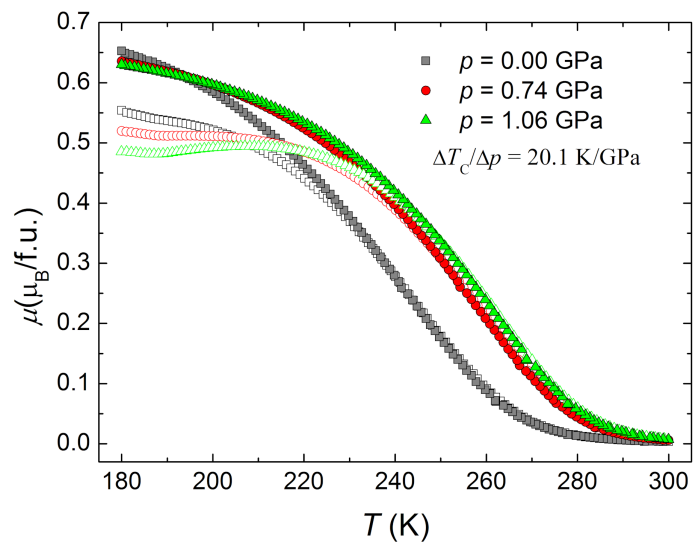

Fig. 4. ZFC and FC magnetization in vicinity of magnetic phase transition are shown at different pressures for sample with rhombohedral crystal structure.

The crystal structure changes from orthorhombic to rhombohedral [5], by annealing at $600^{\circ} \mathrm{C} / 2 \mathrm{~h}$, and $T_{\mathrm{C}}$ increases from $112.0 \mathrm{~K}$ to $245 \mathrm{~K}$ (Fig. 4). Doubling of $T_{\mathrm{C}}$ is mainly due to the increase of bonding angle $\mathrm{Mn}$ O-Mn from 171 to 180 degrees since the strength of DE 


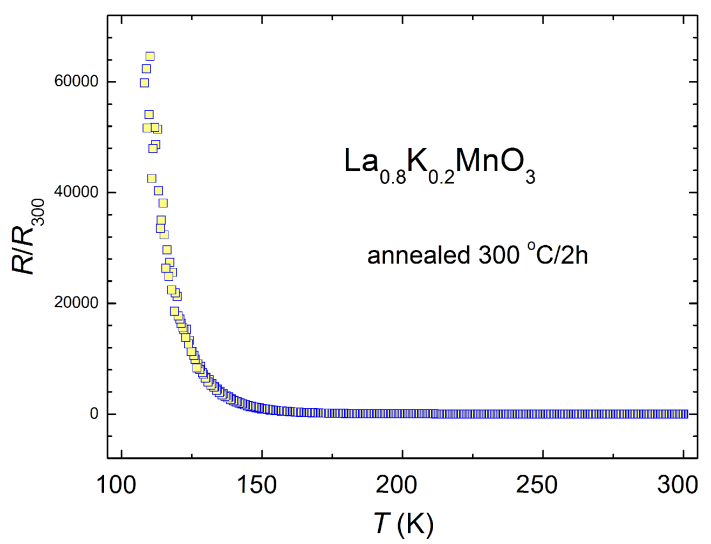

Fig. 5. Temperature dependence of the electrical resistance. The insulator type of resistivity was observed on the sample with the orthorhombic crystal structure.

is proportional to the cosine of this angle [6]. The rhombohedral crystal structure is practically free from JT distortions of lattice, DE interaction is strong and pressure increases $T_{\mathrm{C}}$ with rate $20.1 \mathrm{~K} / \mathrm{GPa}$ (Fig. 4). Pressure experiment can be used as an indirect indicator of JT distortion in such type of material. JT reduces mobility of electrons, which is expected to be high in the case of DE interaction.

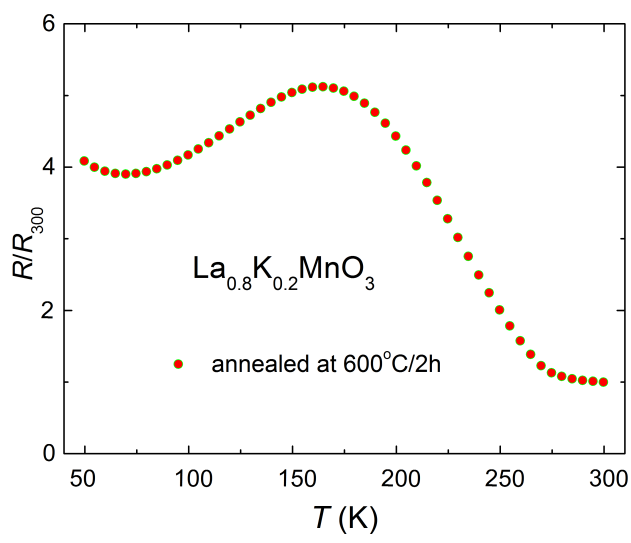

Fig. 6. Temperature dependence of the electrical resistance on the sample with rhombohedral crystal structure.

Measurements of electrical resistivity proof mobility of electrons and can be used as indirect indication of JT distortion in the sample. The insulator type of resistivity was observed on sample with orthorhombic crystal structure which contains JT distortions (Fig. 5). Such a behavior indicates reduction of electron mobility. The insulator-metal transition, which is characteristic feature of hole-doped ferromagnetic manganites with dominant DE interaction, was observed on sample with rhombohedral structure (Fig. 6). At lower temperatures a reentrant transition is present. The JT distortions reduce mobility of electrons and affect the type of electrical resistance in this material.

\section{Conclusions}

Our study of electrical resistance and pressure effect on magnetic properties of the $\mathrm{La}_{0.8} \mathrm{~K}_{0.2} \mathrm{MnO}_{3}$ system of nanoparticles with orthorhombic (space group Pnma) and rhombohedral (space group $R$-3c) crystal structure confirmed theoretical prediction that JT distortion reduces mobility of electrons and DE interaction, which is responsible for ferromagnetic state in this system. Moreover, the reduction of electron mobility due to JT distortion of lattice supports insulator type of resistance.

\section{Acknowledgments}

This work was supported by the project ERDF EU, No. ITMS26220120005, VEGA 2/0132/16 and MAD SKPL 16.

\section{References}

[1] J.M.D. Coey, T. Venkatesan, A.J. Millis, J.R. Cooper, P.C. Riedi, P.B. Littlewood, D.M. Edwards, J.Z. Sun, J. Inoue, Philos. Trans. Math. Phys. Eng. Sci. 365 , 1519 (1998)

[2] A.M. Aliev, A.G. Gamzatov, A.B. Batdalov, A.S. Mankevich, I.E. Korsakov, Physica B 406, 885 (2011).

[3] I.K. Kamilov, A.G. Gamzatov, A.B. Batdalov, A.S. Mankevich, I.E. Korsakov, Phys. Solid State $\mathbf{5 2}$ 789 (2010).

[4] C. Shivakumara, M.B. Bellakki, Bull. Mater. Sci. 32, 443 (2009).

[5] M. Mihalik, M. Zentková, J. Briančin, M. Fitta, M. Mihalik Jr., J. Lazúrová, M. Vavra, Acta Phys. Pol. A 126, 312 (2014)

[6] H. Meskine, S. Satpathy, J. Appl. Phys. 85, 4346 (1999).

[7] D. Markovic, V. Kusigerski, M. Tadic, J. Blanusa, M.V. Antisari, V. Spasojevic, Scr. Mater. 59, 35 (2008).

[8] V. Kusigerski, D. Markovic, V. Spasojevic, M. Tadic, M. Zentková, M. Mihalik, J. Nanopart. Res. 12, 1299 (2010).

[9] P.G. Radaelli, G. Iannone, M. Marezio, H.Y. Hwang, S-W. Cheong, J.D. Jorgensen, D.N. Argyriou, Phys. Rev. B 56, 8265 (1997). 